\title{
Respuesta productiva de gallinas semipesadas inducidas al descanso ovárico en diferentes edades
}

\section{The productive performance of brown egg-laying hens induced to molt at different ages}

\author{
Luis Galeano V, ${ }^{1 *}$ M.Sc, Mónica Estrada $\mathrm{P}^{2}$ M.Sc, Luis Restrepo B, ${ }^{2,3}$ Esp, \\ Juan Sorza Z, ${ }^{3}$ Ph.D.
}

Universidad de Antioquia, Facultad de Ciencias Agrarias, ${ }^{1}$ Grupo Genética, Mejoramiento y Modelación Animal (GaMMA), ${ }^{2}$ Grupo de investigación en avicultura (GIA), ${ }^{3}$ Grupo investigación en Ciencias Agrarias (GRICA), Medellín Colombia. *Correspondencia: gavo76@gmail.com.

Recibido: Octubre de 2010; Aceptado: Marzo de 2012.

\section{RESUMEN}

Objetivo. Evaluar el efecto de la inducción al descanso ovárico (DO) a diferentes edades sobre la respuesta productiva en aves semipesadas productoras de huevo comercial. Materiales y método. Se usaron 840 aves de la línea Hy Line Brown con 64 semanas de edad, distribuidas en 10 tratamientos, consistentes en tres edades $(65,70$ y 75 semanas) con la aplicación de tres períodos de ayuno $(5,10$ y 15 días) y un control (sin DO). El análisis estadístico utilizó un modelo completamente aleatorizado, anidado, efecto fijo y balanceado. Se evaluó consumo de calcio durante el ayuno ( $\mathrm{g} / \mathrm{ave} / \mathrm{d} i ́ \mathrm{a})$, consumo de alimento ( $\mathrm{g} / \mathrm{ave} /$ día), porcentaje de producción, peso del huevo $(\mathrm{g})$, conversión por masa de huevo y huevos por ave alojada (HAA). Resultados. La edad presentó efecto significativo $(p<0.05)$ sobre las variables consumo de alimento, día del primer huevo postmuda, días sin producción, día postmuda de retorno al $50 \%$ de producción, peso del huevo, número de huevos, masa huevos, conversión y porcentaje de producción $(\mathrm{p}<0.01)$. Al comparar con el control, el tratamiento $75-5$ presentó efecto significativo $(p<0.05)$ para las variables: consumo de alimento, número de huevos promedio, masa huevos y conversión. Conclusiones. Las aves con mayor período de ayuno presentaron mayor período improductivo postmuda, grandes pérdidas de peso y alto consumo de alimento, lo que genera alta conversión e ineficiencia productiva. En comparación con reportes de literatura sobre DO en aves livianas, las aves semipesadas llegan al cese de producción en menos días y su período improductivo es menor.

Palabras clave: Ayuno, consumo de alimentos, edad, producción de huevos (Fuente:AGROVOC). 


\begin{abstract}
Objective. The application of the ovarian rest technique on brown egg layer birds was evaluated in this investigation as well as the effect of age and feed duration withdrawal have over their productive behavior. Materials and method. $840 \mathrm{Hy}$ Line Brown hens at 64 weeks of age, evaluating three ages $(65,70$ and 75 weeks) and three feed withdrawal periods $(5,10$ and 15 days) in a completely randomized, nested, fixed and balanced effects model was used. The calcium consumption during feed withdrawal ( $\mathrm{g} / \mathrm{bird} / \mathrm{day}$ ), percentage of production, egg weight $(\mathrm{g})$, conversion per egg mass and eggs per housed hen were evaluated. Results. The age factor presented a significant effect $(p<0.05)$ for food consumption, day of the first egg laid post-molting, days without production, day of return to $50 \%$ of the production post-molting, egg weight, number of eggs average, egg mass, conversion and $(p<0.01)$ production percentage. After comparing the control treatments, the $75-5$ treatment presented a significant effect $(p<0.05)$ for food consumption, average number of eggs, egg mass and conversion. Conclusions. Birds with a greater feed withdrawal period presented a greater unproductive post-molting period, greater weight loss and greater consumption of food which reflects on high conversion and could be defined as productive inefficiency. The comparison between lines showed that cease of production.
\end{abstract}

Key words: Age, egg production, feed intake, feed restriction (Source:AGROVOC).

\section{INTRODUCCIÓN}

En la producción avícola, el ave a mayor edad tiende a disminuir su nivel productivo de huevos, tanto en cantidad como en calidad hasta el punto que económicamente no es viable para el productor $(1,2)$. Esta evolución natural de los rendimientos productivos del ave, puede ser alterada por el productor utilizando el descanso ovárico (DO).

EI DO es una técnica adaptada del comportamiento natural de las aves, donde entran en período de ayuno y cambio de plumaje durante las estaciones de invierno, los períodos de incubación o migración (3).

La efectividad del DO depende de la capacidad de generar el nivel de estrés óptimo en el ave, que desencadene alteraciones hormonales y que tenga como resultado el cese de la producción de huevos. Autores como Etches (1984), Williams (1985) citados por Kretzschmar et al (1) y Berry (4), insisten en la importancia del aumento de la corticosterona y la disminución de LH (hormona luteinizante), el cese de postura y la pérdida de peso como indicadores de la eficacia del DO (1). Para obtener este efecto en el ave se usan estímulos externos inductores al DO, tales como: la restricción de la alimentación, la manipulación del fotoperiodo, la inclusión de desbalances nutricionales, el suministro de hormonas y de sustancias químicas entre otros $(5,6)$.

Con respecto al cese de la producción, se ha considerado necesario para la regeneración del aparato genital $(4,7)$ y rejuvenecimiento de la zona reproductiva (8-10), permitiendo la recuperación en el nivel productivo, no tan alto como el ciclo anterior, pero con un peso de huevo promedio mayor y una calidad de cáscara óptima $(3,5,11)$. En resumen, la técnica del DO se utiliza para ayudar a manejar y sincronizar los ciclos de la producción de un lote de aves $(1,2$,$) ; debido a que durante la muda$ suspenden la producción de huevos, pero luego de ser nuevamente estimuladas, retornan a la producción igualando o superando la respuesta productiva antes del estimulo inductor del cese productivo, este segundo ciclo puede durar entre 25 a 35 semanas $(11,12)$.

La aplicación del DO, parte de la evaluación de criterios tales como: la disminución en el nivel de producción, la alteración en la calidad externa e interna del huevo y el aumento de los costos en la crianza de lotes de reemplazo, entre otros (13).

El objetivo de este trabajo fue caracterizar y documentar la respuesta productiva de la aplicación de la técnica del descanso ovárico con restricción alimenticia, con duración de cinco, diez y quince días, en aves semipesadas de 65, 70, 75 semanas de vida.

\section{MATERIALES Y MÉTODOS}

Sitio de estudio. La investigación se llevo a cabo en la granja productora de huevo comercial Aves Emaús, localizada en el municipio de El Retiro (Antioquia), noroccidente de Colombia, a 2400 m.s.n.m. y con temperatura promedio de $18^{\circ} \mathrm{C}$. 
Muestra y tratamiento. Fueron seleccionadas 840 gallinas de la linea Hy Line Brown ${ }^{\circledR}$ con 64 semanas de edad con un peso promedio $(1930 \pm 95 \mathrm{~g})$, acorde a los pesos propuestos en la guía de manejo de la casa comercial para la edad.La evaluación productiva se realizó hasta la semana 100 de vida de las aves. Las aves se distribuyeron aleatoriamente en jaulas, cada una con dimensión de $35 \times 45 \mathrm{~cm}$, garantizando 525 $\mathrm{cm}^{2}$ por ave. A cada jaula se le asignó al azar uno de los 10 tratamientos combinando las tres edades $(65,70$ y 75 semanas de vida) y tres períodos de ayuno $(5,10$ y 15 días $)$.

El tratamiento control no recibió ningún estímulo inductor del cese productivo (DO). Cada unidad experimental correspondió a dos jaulas contiguas (seis aves por réplica) y catorce réplicas por tratamiento. Las aves recibieron en forma continua agua fresca y tratada en bebederos de copa. Durante los períodos de ayuno evaluados, se suministró calcio a voluntad en presentación de grit. El método de realimentación postayuno consistió en la oferta gradual, iniciando $30 \mathrm{~g} / \mathrm{ave}$ el primer día, $60 \mathrm{~g} / a v e$ el segundo día, $90 \mathrm{~g} / \mathrm{ave}$ el tercero y a partir del cuarto día se entregó el alimento a voluntad. La composición de la dieta postmuda proporcionada a las aves se elaboró con base en lo propuesto por Koelkebeck (14).

Recolección de huevos. Se hizo diariamente, y los datos generados se consignaron en un registro de producción para cada réplica. Esta información se procesó y se utilizó para el cálculo del porcentaje de producción de huevos por réplica, el período improductivo de cada uno de los tratamientos, día postayuno de inicio del cese producción, día postayuno de retorno a la producción y el tiempo que tardaron los tratamientos para llegar al $50 \%$ de producción. Para la determinación de la conversión en masa de huevo, se pesaron los huevos de todos los tratamientos un mes después de terminado el ayuno y se repitió por cuatro veces durante el período restante hasta la semana 100 de evaluación.

Grupo de aves. Las aves utilizadas para la evaluación estaban divididas en tres grupos, aves en evaluación, aves para sacrificio y aves denominadas reemplazo. Los tres grupos se distribuyeron en los distintos tratamientos y recibieron el mismo manejo, lo que permitía que si un ave moría en el grupo de evaluación era inmediatamente reemplazada por otra que hubiese recibido el mismo período de ayuno del grupo reemplazo, garantizando seis aves por réplica durante todo el seguimiento. Esta información se utilizó para la creación de un registro por réplica de las aves que morían y eran reemplazadas para poder calcular la mortalidad de la aplicación del DO.

Análisis estadístico. Se utilizó un diseño experimental completamente aleatorizado, anidado, de efecto fijo balanceado con catorce réplicas por tratamiento, anotando que el factor ayuno se anidó en el factor edad, por lo tanto no existe interacción entre dichos factores. De ahí que el tratamiento control se comparaba dentro de cada condición de anidamiento, generando el siguiente modelo:

$Y_{\mathrm{ijk}}=\mu+a_{\mathrm{i}}+\beta_{\mathrm{j}(\mathrm{i})}+\varepsilon_{\mathrm{k}(\mathrm{ij})}$

Donde:

$Y_{i j k}$ : Variable de respuesta

$\mu$ : Efecto promedio general

$a_{i}$ : Efecto de la i-ésima edad

$\beta_{\mathrm{j}(\mathrm{i})}$ : Efecto del j-ésimo período de ayuno anidado en la i-ésima edad

$\varepsilon_{\mathrm{k}(\mathrm{j})}$ : Error experimental asociado al modelo.

Se empleó la prueba de contraste de Tukey, a fin de comparar el efecto promedio de los tratamientos dentro de cada condición asociada con el diseño anidado. Se efectuó un análisis descriptivo unidimensional para las variables: Consumo de calcio, consumo de alimento, peso del huevo, porcentaje de producción de huevos utilizando el software SAS v 8.2 (15).

Al efectuar el análisis de la variable porcentaje de producción, no se cumplió con el supuesto de normalidad en los términos del error experimental, por lo que se procedió a transformar los datos mediante el empleo de la familia de transformación Box Cox (16), donde se obtuvo por máxima verosimilitud el lambda, que al aplicar a los datos no logró hacer cumplir el supuesto de normalidad. Por tal motivo, se modificó el alpha a un nivel del $1 \%$ para efectuar los ajustes y así mitigar el efecto de inflación que presenta el error tipo I.

\section{RESULTADOS}

El efecto de la aplicación del DO para la variables consumo de calcio no fue significativo $(p>0.05)$ para las edades evaluadas; pero para el consumo de alimento, esta variable presentó efecto significativo $(p<0.05)$ para la edad y tiempo de ayuno dentro de cada nivel de edad. Siendo la edad a las 75 semanas en la que se obtuvo el más alto consumo, en comparación con los demás niveles del factor y los referentes teóricos de la casa genética de la línea Hy lyne Brown ${ }^{\circledR}(17)$; el menor consumo se presentó en el tratamiento control (Tabla 1). 
Tabla 1. Efecto de la edad de aplicación del DO en el consumo de calcio y alimento.

\begin{tabular}{ccc}
\hline \multirow{2}{*}{ Factor Edad (sem) } & \multicolumn{2}{c}{ Consumo (g/ave/día) } \\
\cline { 2 - 3 } & Calcio & Alimento \\
\hline \multirow{2}{*}{65} & $12.178^{\mathrm{a}}$ & $111.711 \mathrm{~b}$ \\
& \pm 7.198 & \pm 6.86 \\
70 & $12.307 \mathrm{a}$ & $115.509 \mathrm{~b}$ \\
& \pm 6.967 & \pm 9.05 \\
75 & $17.667 \mathrm{a}$ & $128.913 \mathrm{a}$ \\
& \pm 10.629 & \pm 3.45 \\
& & \\
Modelo* & NS & $* * *$ \\
E Sign & NS & $* * *$ \\
A(E) Sign & NS & $* * *$ \\
\hline
\end{tabular}

Letras diferentes en una misma columna indica diferencia significativa *** $(p<0.05) . E=E d a d, N S=$ No significativo y $A(E)=$ Ayuno anidado en la edad

En la tabla 2, se puede observar como el modelo presentó efecto significativo para todas las variables evaluadas $(\mathrm{p}<0.05)$ y altamente significativo $(p<0.01)$ para la variable porcentaje de producción, a excepción de la variable mortalidad.

Tabla 2. Efecto de la edad y duración del ayuno en la aplicación del DO sobre la producción y conversión.

\begin{tabular}{|c|c|c|c|c|}
\hline $\begin{array}{l}\text { Factor Edad } \\
\quad(\text { sem })\end{array}$ & $\begin{array}{c}\text { Peso } \\
\text { huevo } \\
\text { (g) }\end{array}$ & $\begin{array}{l}\text { Número } \\
\text { de huevos } \\
\text { promedio }\end{array}$ & $\begin{array}{c}\text { Masa } \\
\text { huevo } \\
\text { (g) }\end{array}$ & Conversión \\
\hline 65 & $\begin{array}{l}70.25^{\mathrm{a}} \\
\pm 4.33\end{array}$ & $\begin{array}{l}257.56^{a} \\
\pm 65.89\end{array}$ & $\begin{array}{l}18056^{a} \\
\pm 4506\end{array}$ & $\begin{array}{c}3.32^{\mathrm{b}} \\
\pm 0.81\end{array}$ \\
\hline 70 & $\begin{array}{l}71.58^{a} \\
\pm 3.69\end{array}$ & $\begin{array}{l}198.72^{\mathrm{b}} \\
\pm 67.23\end{array}$ & $\begin{array}{l}14225^{b} \\
\pm 4813\end{array}$ & $\begin{array}{l}4.55^{\mathrm{ab}} \\
\pm 1.51\end{array}$ \\
\hline 75 & $\begin{array}{l}71.09^{a} \\
\pm 4.90\end{array}$ & $\begin{array}{l}191.94^{b} \\
\pm 80.14\end{array}$ & $\begin{array}{l}13712^{b} \\
\pm 5913\end{array}$ & $\begin{array}{l}5.77^{a} \\
\pm 2.84\end{array}$ \\
\hline Modelo* & $* * *$ & $* * *$ & $* * *$ & $* * *$ \\
\hline E Sign & NS & $* * *$ & $* * *$ & $* * *$ \\
\hline$A(E)$ Sign & $* * *$ & $* * *$ & $* * *$ & $* * *$ \\
\hline
\end{tabular}

El factor edad presentó efecto significativo $(p<0.05)$ en las variables: primer huevo postmuda, días sin producción, día postmuda de retorno al $50 \%$ de producción y altamente significativo $(p<0.01)$ para la variable porcentaje de producción. El factor de período de ayuno anidado dentro del factor edad, presentó efecto significativo $(p<0.05)$ para todas las variables anotando alta significancia $(\mathrm{p}<0.01)$ para la variable porcentaje de producción.

Para el día de inicio del cese de producción, no se encontró efecto significativo de la edad, ni diferencia entre edades ( $p>0.05$; Tabla 3). Lo que sugiere que, sin importar la edad a la que se aplique de inducción del DO o la duración del ayuno, el ave cesa de producir en promedio de 5 a 6 días de iniciado el estímulo.
Tabla 3. Variables para valoración de la efectividad productiva del DO

\begin{tabular}{|c|c|c|c|c|c|c|}
\hline FE & DCP & PHP & DSP & DP\% & $\% \mathrm{M}$ & \% P \\
\hline 65 & $\begin{array}{c}5.777 a \\
\pm 218\end{array}$ & $\begin{array}{c}10.111 b \\
\pm 3.78\end{array}$ & $\begin{array}{c}13.944 b \\
\pm 6.74\end{array}$ & $\begin{array}{c}13.055 b \\
\pm 2.20\end{array}$ & $\begin{array}{c}24.07 a \\
\pm \\
29.82\end{array}$ & $\begin{array}{c}50.810 b \\
\pm 4.33\end{array}$ \\
\hline 70 & $\begin{array}{l}5.944 a \\
\pm 1.98\end{array}$ & $\begin{array}{c}12.277 a \\
\pm 1.74\end{array}$ & $\begin{array}{c}16.444 a \\
\pm 4.50\end{array}$ & $\begin{array}{c}14.833 b \\
\pm 2.57\end{array}$ & $\begin{array}{c}20.37 a \\
\pm \\
28.32\end{array}$ & $\begin{array}{c}39.195 b \\
\pm 3.69\end{array}$ \\
\hline 75 & $\begin{array}{l}6.500 a \\
\pm 1.46\end{array}$ & $\begin{array}{c}13.000 a \\
\pm 4.47\end{array}$ & $\begin{array}{c}16.611 \mathrm{a} \\
\pm 7.95\end{array}$ & $\begin{array}{c}17.333 a \\
\pm 5.80\end{array}$ & $\begin{array}{c}24.07 a \\
\pm \\
27.54\end{array}$ & $\begin{array}{c}37.080 b \\
\pm 4.90\end{array}$ \\
\hline Mod & $* * *$ & $* * *$ & $* * *$ & $* * *$ & NS & $* * * *$ \\
\hline $\begin{array}{c}E \\
\text { Sign }\end{array}$ & NS & $* * *$ & $* * *$ & $* * *$ & NS & $* * * *$ \\
\hline $\begin{array}{l}A(E) \\
\text { Sign }\end{array}$ & $* * *$ & $* * *$ & $* * *$ & $* * *$ & NS & $* * * *$ \\
\hline
\end{tabular}

FE: Factor Edad (sem); DCP: Día de inicio cese producción; PHP: Primer huevo postmuda; DSP: Días sin producción; DP\%: Día postmuda de retorno al 50\% de producción; \%M: \% Mortalidad; Letras diferentes en una misma columna indica diferencia significativa. **** Altamente significativo $(p<0.01), * * *$ Significativo $(p<0.05), E=E d a d, A(E)=$ Ayuno anidado en la edad y NS= No significativo

La mortalidad no presentó efecto significativo ( $p>0.05$ ) para los factores edad y duración del ayuno anidado dentro del factor edad, lo que indica que las aves muertas en la evaluación no fueron consecuencia directa de la edad en que se aplicó el estímulo o la duración del período de ayuno; de hecho las aves murieron durante todo el período de evaluación, incluyendo el tratamiento control que no recibió ningún estímulo para la inducción al DO.

La variable porcentaje promedio de producción de huevos (Tabla 3), presentó efecto altamente significativo $(p<0.01)$ para el modelo, la edad de aplicación del DO y el período de ayuno. Para la edad 65 se encontró el mayor porcentaje promedio de producción, pero sin diferencia significativa $(p>0.05)$ con las edades 70 y 75 . En las figuras 1,2 y 3 se presentaron los picos de producción por tratamiento siendo el tratamiento a la edad de 65 semanas y con un tiempo de ayuno de 10 días el que obtuvo el mayor nivel productivo.

Las variables productivas peso del huevo, número de huevos promedio, masa de huevos y conversión presentaron efecto significativo $(p<0.05)$ para las fuentes de variación tenidas en cuenta en el modelo experimental, para el factor edad se encontró efecto significativo $(p<0.05)$ sobre las variables número de huevos, masa de huevos y conversión, pero no significativo para el peso del huevo ( $p>0.05)$ (Tabla 2$)$.

Al evaluar las variables productivas porcentaje promedio de producción (Tabla 3 ) y el número de huevos (Tabla 2), la primera presenta diferencia altamente significativa $(p<0.01)$ entre las edades, mientras que la segunda, presenta 
diferencia estadística $(p<0.05)$ entre la edad 65 con respecto a las edades 70 y 75 . Por este mismo efecto, tanto la masa de huevo como la conversión presentan el mismo comportamiento de las variables porcentaje de producción y número de huevos.

En el caso de la conversión, entendida como el indicador de la eficiencia del proceso, toma mayor importancia al ser valorada económicamente y debe ser tenida en cuenta como referente para la decisión de la aplicación o no del DO. La tabla 2 muestra una mayor ineficiencia a medida que aumenta la edad, esto como consecuencia del mayor consumo de alimento y a la menor producción reportada por las aves, indicando una posible mayor rentabilidad el proceso en edades tempranas.

Comparación pareada entre los tratamientos y el control. Con el fin de contrastar las variables consumo alimento (g/ave/día), peso del huevo (g), número de huevos promedio, masa huevo (g), conversión y huevos por ave alojada (HAA) dentro de cada condición de anidamiento con el control, fue necesario tomar en cuenta que todos presentaron curvas de producción en el tiempo; por tal motivo se decidió comparar un período de 169 días para cada tratamiento con el mismo período de tiempo para tratamiento control.

La variable consumo de alimento para las condiciones 70-15, 75-5, 75-10 y 75-15, presentó diferencia significativa $(p<0.05)$ con el tratamiento control.

El peso del huevo presentó efecto significativo $(p<0.05)$ para el tratamiento $65-10$ con respecto al control.

En lo que respecta a la producción promedio de huevos del segundo ciclo, se presentó diferencia significativa $(p<0.05)$ entre el tratamiento 70-15 y el control, y entre el tratamiento 75-5 y el control.

La variable masa de huevo $(\mathrm{g})$ sólo presentó efecto significativo $(p<0.05)$ para el tratamiento $75-5$ y el control. De forma similar, la variable conversión mostró efecto significativo $(p<0.05)$ para el tratamiento 75-5 y el control. Ambas variables están condicionadas por el resultado del número de huevos y su peso, con respecto a esto el tratamiento 75-5 fue el de mayor diferencia de HAA con respecto al control (40-45).

\section{DISCUSIÓN}

Para la valoración de la efectividad productiva del método de inducción al DO se determinaron variables o indicadores, tales como: día de inicio del cese de producción después de iniciado el ayuno, día del primer huevo postmuda, duración en días del período sin producción, día postmuda de retorno al 50\% de producción y porcentaje de mortalidad (Tabla 2); ya que Bell(18) considera que un buen método es aquel que pueda provocar la situación de estrés precisa para interrumpir la producción de huevos de forma rápida y homogénea en el lote, minimizando así, el período improductivo de las aves y lograr alcanzar rápidamente los niveles productivos rentables (más del $50 \%$ del lote), con una mortalidad baja y una alta producción en el siguiente ciclo o como mínimo igualando la producción del primer ciclo.

La edad del ave influyó en la ineficiencia productiva, ya que presentó una caída de la producción a medida que la edad del ave aumentó. Con respecto a la edad de aplicación del DO, no importa la edad a la que se aplique el estímulo de inducción del DO, siempre que se someta el ave a un período de ayuno igual o superior a 5 días el ave cesa de producir en promedio de 5 a 6 días de iniciado el estímulo. Para la variable: consumo de alimento, ésta mostró una tendencia a un mayor consumo de alimento a medida que aumenta la edad del ave; indicando que el efecto de recuperación del ave, como consecuencia del ayuno, parte del aumento en el consumo de alimento y el consecuente aumento de peso y posterior retorno a la producción de huevos.

Al comparar los tratamientos con el control este último presentó un consumo inferior al tratamiento con el que se comparó; reafirmando lo expresado con el análisis, donde las aves que fueron sometidas al DO a mayor edad tienen mayor consumo de alimento y con ello, mayor ineficiencia productiva (mayor conversión). Este comportamiento se puede considerar como una de las adaptaciones del ave en respuesta a la pérdida de peso corporal ocasionada por el ayuno y la posterior recuperación con respecto a las no sometidas al DO (11).

El desempeño productivo de las aves en el segundo ciclo, se comienza a evaluar con los días improductivos a partir de la aplicación del DO, lo cual indica que el período improductivo aumenta a medida que el ave envejece, que bien podría interpretarse como una menor velocidad de recuperación del tracto reproductivo del ave a medida que aumenta la edad, aspecto expresado por Bar (19), quien recomienda no realizar inducciones al DO en aves con una avanzada edad, ojalá con menos de 100 semanas de vida.

En los tratamientos que no presentaron diferencia significativa en lo que respecta a la producción 
promedio de huevos del segundo ciclo, al igual que Webster, (20) entre aves con distintos niveles de pérdida de peso, e incluso con aquellas que no recibieron el estímulo inductor al DO. Lo que demuestra que períodos largos de ayuno como inductores al DO tienen efecto en la respuesta productiva del ave, pues aunque generan mayor período improductivo, logran igualar el promedio de huevos de las aves con menor período improductivo; no obstante cabe anotar que ésta ventaja productiva no arrojó diferencia en cantidad de huevos promedio con aplicación o no del DO.

La producción de huevos presentó una alteración en la curva, esto pudo ser consecuencia del desgaste productivo del ave a medida que envejece. Conclusión que se asume con base en los resultados obtenidos por Galeano (21), donde el porcentaje de LFY (porcentaje de folículos con un diámetro igual o superior a $10 \mathrm{~mm}$ ), mostró tendencia a la disminución a medida que aumentaba la edad, lo que demuestra que las aves no generaron la respuesta esperada de persistencia productiva en el segundo ciclo propuesta por otros autores. Pero esta disminución no se presentó en todos los tratamientos, además no fue una caída gradual en los tratamientos que manifestaron la alteración productiva; por tal motivo, queda como interrogante para nuevas investigaciones que permitan determinar si el nivel óptimo productivo para la línea de aves evaluadas sólo puede llegar hasta la semana 95 de vida o si ésta disminución en la respuesta productiva de las aves sólo fue un caso marcado por el carácter biológico de los animales evaluados.

Con respecto a esto, McDaniel (12) sustenta que la aplicación del DO puede aumentar el período de producción de 25 a 35 semanas en el ciclo postmuda, pero como se puede ver en las figuras de producción, en el caso de la semana 75 de aplicación del DO, contó con 12 ó 13 semanas de producción antes de llegar a menos del $10 \%$ de producción, en contraste con 16 a 19 semanas para la edad 70, y 25 a 27 semanas para la edad 65. Por esta variación en la duración del período productivo postayuno entre los tratamientos, fue necesario para el análisis de la producción delimitar un lapso similar para los tratamientos de 169 días a partir de la finalización del ayuno.

La variable peso del huevo indicó que el peso del huevo no depende de la edad, ya que en condiciones normales, el tamaño y el peso del huevo tienden a aumentar después del pico de producción, debido al incremento del peso corporal del ave y la disminución en el nivel de producción $(5,8,22)$. Todos los pesos obtenidos en el experimento fueron mayores a los datos reportados en la guía de manejo de la línea para las mismas edades (17), lo que confirma lo propuesto por Flemming(22) y Webster(20), quienes afirmaron que una de las ventajas de la utilización del DO es el aumento en el tamaño del huevo postmuda (Tabla 2 ). Otra posible causa por lo que la edad no tuvo efecto en el que al peso del huevo se refiere, pudo ser por el período tan corto de cinco semanas entre las edades evaluadas y el corto período de evaluación de 169 días.

Estadísticamente la comparación de los efectos promedios de los tratamientos y el control no presentaron diferencia significativa $(p>0.05)$ con respecto a la variable número de huevos producidos promedio. Este resultado fue consecuencia de la comparación de los porcentajes de producción entre el control y los tratamientos que presentaban semanas postayuno con producciones bajas, lo provocó la disminución en el valor promedio. También es de anotar, las desviaciones estándar son amplias entre los tratamientos comparados con el control, hasta de 80 huevos. Por lo cual, ésta variable no sirvió como indicador de la efectividad del DO en la producción.

Por consiguiente, la valoración productiva del DO se hizo a partir de la comparación de la HAA entre los tratamientos y el control.

Se encontró cantidades de huevos diferentes entre las edades y el control, también se observó la tendencia a la disminución de la cantidad de huevos a medida que envejece el ave tanto para la edad evaluada como para el control, además, la diferencia a favor de las edades con respecto al control y el aumento de ésta diferencia en relación al aumento de la edad del ave. Esto se explica a partir de los rendimientos decrecientes, donde normalmente el ave tiende a disminuir su productividad al aumentar la edad, pero al ser sometida al DO, la producción llega a cero retornando al segundo ciclo productivo con el pico de producción que supera los niveles productivos de la curva normal del ave que no es inducida al DO (Figuras 1,2 y 3 ).

En la comparación de los tratamientos con el control se apreció que no hay una tendencia clara en lo que respecta a la duración del período de ayuno y el número de huevos por ave para cada edad.

Comparación productiva entre aves livianas y semipesadas. Para el análisis, se tuvo en cuenta que los resultados en las investigaciones de aves livianas provienen de la aplicación del DO, a partir 


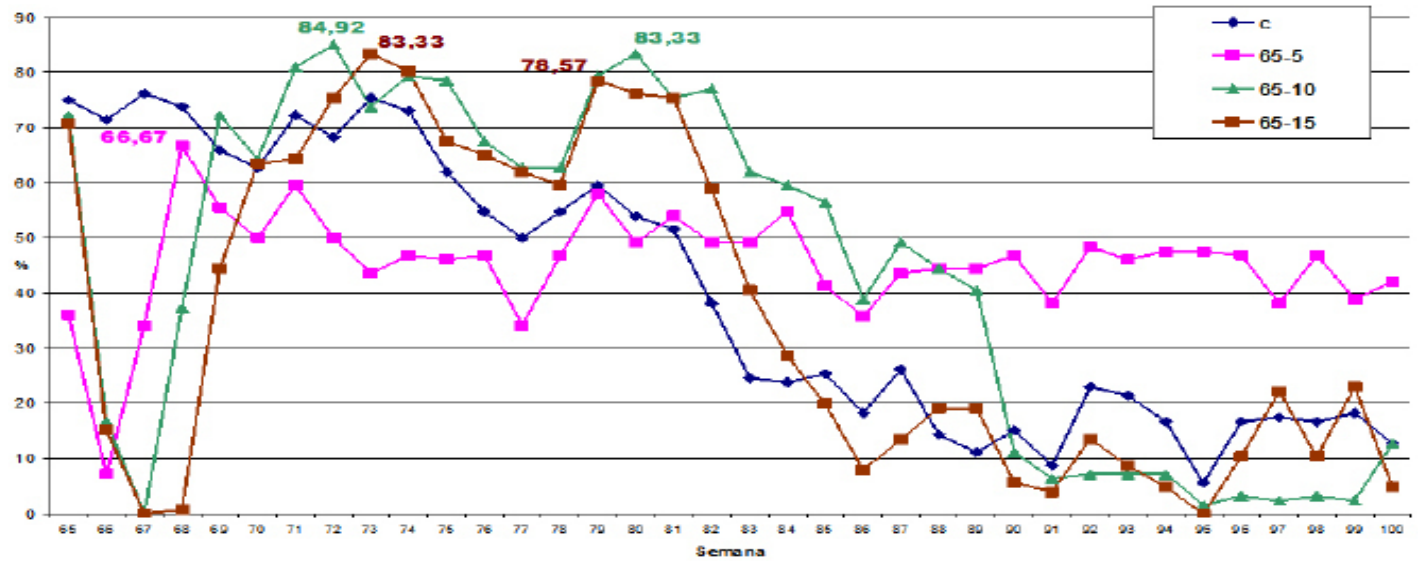

Figura 1. Curvas de producción para los tratamientos de la edad 65.

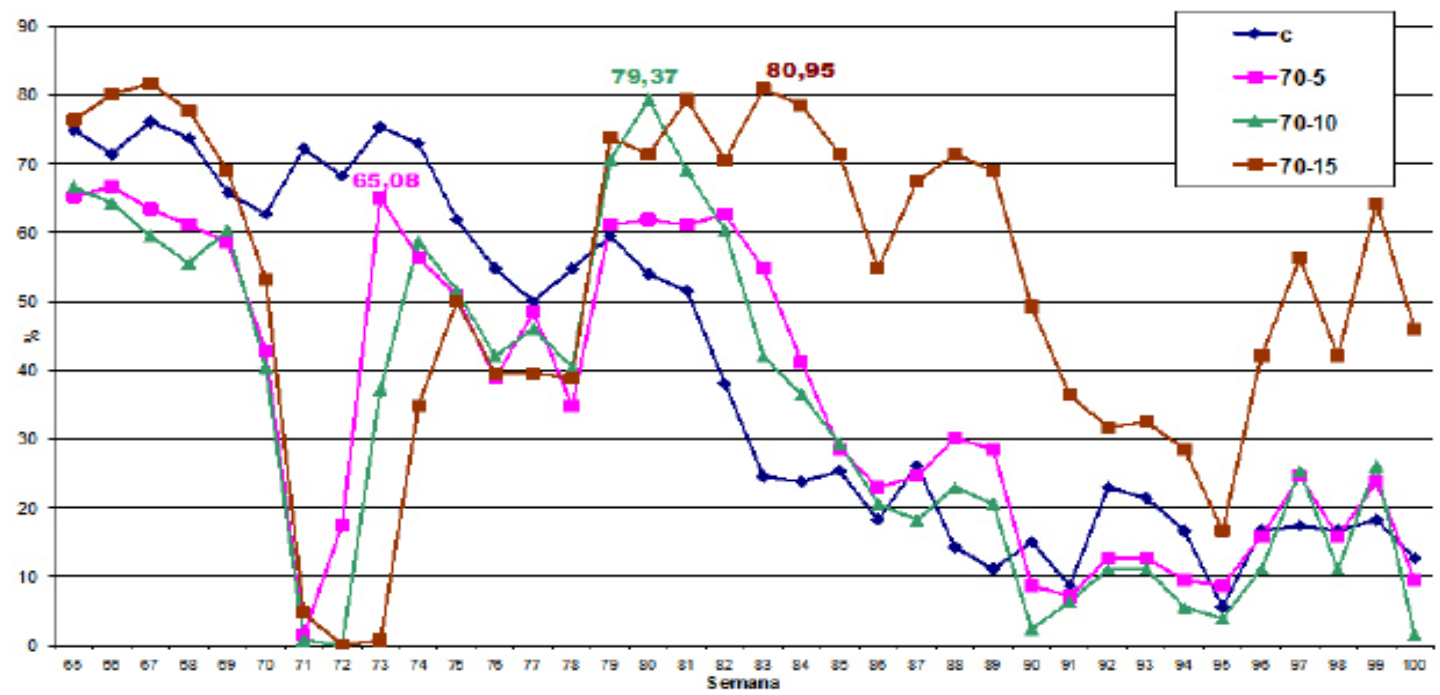

Figura 2. Curvas de producción para los tratamientos de la edad 70.

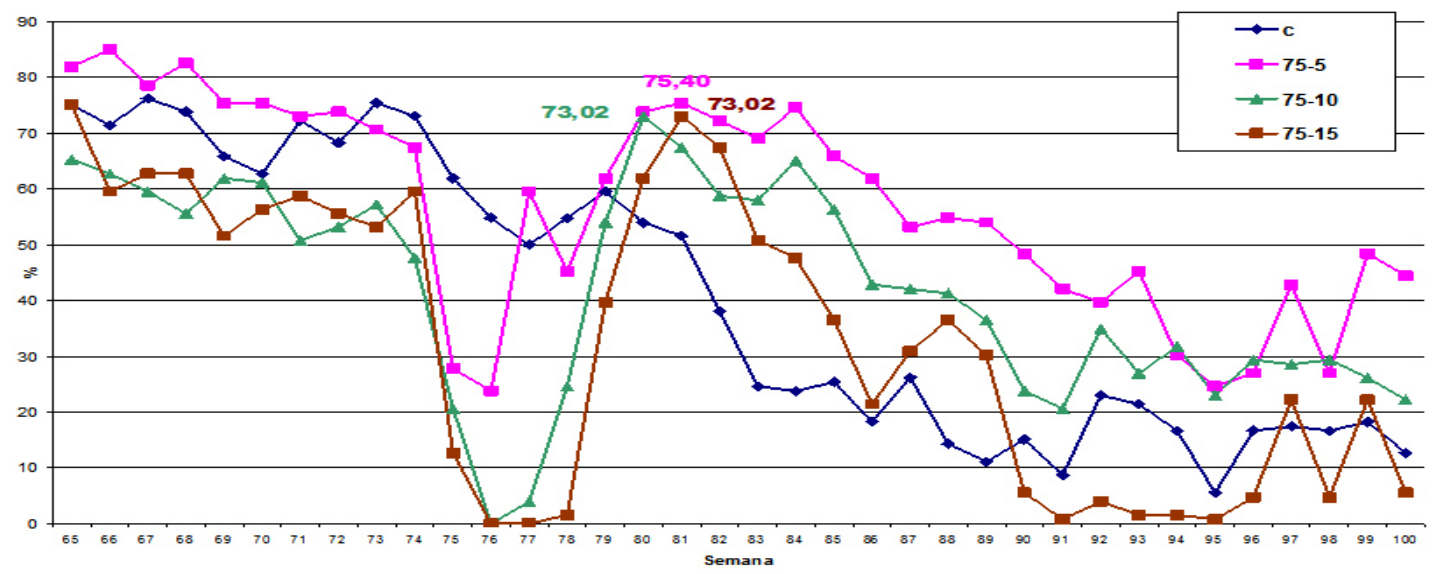

Figura 3. Curvas de producción para los tratamientos de la edad 75. 
de la utilización como estímulo inductor el ayuno y la manipulación del fotoperiodo, mientras que los resultados de esta investigación sólo tuvieron en cuenta la utilización del ayuno como estímulo inductor del DO.

Aun así, la comparación de los resultados mostró que el cese de la producción se presenta en menos días en aves livianas que en aves semipesadas y el período improductivo o período de recuperación del tracto reproductivo de las aves de líneas livianas es mayor al presentado por las aves en el ensayo. Lo que comparativamente ofrece al ave semipesada un menor período improductivo y una mayor capacidad de recuperación al ayuno.

Con respecto a la mortalidad, para las aves del experimento es mayor ( 20 a $25 \%$ ), comparado con el $2 \%$ al $6 \%$ reportado para aves livianas, pero en las publicaciones no especifican si el dato reportado corresponde a las aves que murieron en todo el período de evaluación o únicamente durante la aplicación del estímulo inductor al DO; tampoco se hace claridad si la muerte del ave se presentó como consecuencia a la técnica de inducción al DO o por otros motivos. En el caso de la evaluación, el dato de mortalidad reportado corresponde a la totalidad del período de seguimiento y no está relacionado, ni con la edad del ave, el período de ayuno o el método de inducción al DO.
Esta comparación, permite mostrar la gran diferencia existente entre la respuesta de aves de líneas livianas y semipesadas al ser sometidas al DO utilizando el ayuno como efecto inductor; lo que requiere ahondar en la diferenciación de las alternativas de inducción al DO y su aplicación en las distintas líneas de aves, y en especial, en el metabolismo lipídico del ave semipesada, como fuente energética y precursor hormonal (23), como posible gran diferenciador de su comportamiento en comparación con las aves de líneas livianas.

Queda como objetivos de próximos trabajos la identificación el efecto del DO sobre las reservas lipídicas del ave semipesada y el papel metabólico que cumplen en el ave en períodos de ayuno, así también, valorar cual es la edad productiva a la cual puede llegar un ave. Otro aspecto a tener en cuenta para futuras investigaciones, es el desarrollo de una estructura de costos para el análisis microeconómico y la valoración del efecto de la edad y la duración del período de ayuno en la respuesta productiva del DO.

En conclusión, con base en el análisis de graficas de producción, una inducción tardía del DO, permitiría tener un margen superior de huevos al compararlos con una curva normal de producción, que normalmente estaría en proceso de finalización. Esta diferencia entre ambas curvas de producción debería ser significativa para hacer de la inducción al DO una alternativa viable.

\section{REFERENCIAS}

1. Moore RW, Holt PS. The Effect of Feed Deprivation on Tissue Invasion by Salmonella enteritidis. Poult Sci 2006; 85:1333-1337.

2. Kretzschmar-McCluskey $V$, Curtis PA, Anderson KE, KerthLK, Berry WD. Influence of Hen Age and Molting Treatments on Shell Egg Exterior, Interior, and Contents Microflora and Salmonella Prevalence. During a Second Production Cycl. Poult Sci 2008; 87:2146-2151.

3. Dunkley CS, Friend TH, McReynolds JL, Woodward CL., Kim WK, Dunkley KD, Kubena F, Nisbet DJ,Ricke S. C. Behavioral Responses of Laying Hens to Different Alfalfa-Layer Ration Combinations Fed During Molting. Poult Sci 2008; 87:1005-1011.
4. Berry WD. The physiology of induced molting. Poult Sci 2003; 82: 971-980.

5. Koch JM, Moritz JS, Smith DL, Lay DC, Wilson ME. Melengestrol Acetate as an Effective Alternative to Induce a Decline in Egg Production and Reversible Regression of the Reproductive Tract in Laying Hens II. Effects on Postmolt Egg Quality. Poult Sci 2005; 84:1757-1762.

6. Kubena LF, Byrd JA. Effects of drinking water treatment on susceptibility of laying hens to Salmonella enteritidisduring forced molt. Poult Sci 2005; 84:204-211.

7. Mazzuco $\mathrm{H}$, Hester PY. The effect of an induced molt and a second cycle of lay on skeletal integrity of White Leghorns. Poult Sci 2005; 84:771-781. 
8. Wu G, Gunawardana P, Bryant MM, Voitle RA, Roland Sr DA. Effect of Molting Method and Dietary Energy on Postmolt Performance, Egg Components, Egg Solid, and Egg Quality in Bovans White and DekalbWhite Hens During Second Cycle Phases Two and Three. Poult Sci 2007; 86:869-876.

9. Mazzuco $\mathrm{H}$, Hester PY. The Effect of an Induced Molt Using a Nonfasting Program on Bone Mineralization of White Leghorns1. Poult Sci 2005; 84:1483-1490.

10. Szabo A, Fe' bel H, Me 'zes M, Horn P, Balogh K, Romva'ri R. Differential Utilization of Hepatic and Myocardial Fatty Acids During Forced Molt of Laying Hens. Poult Sci 2005; 84:106-112.

11. Webster AB. Physiology and behavior of the hen during induced molt. Poult Sci 2003; 82:992-1002.

12. McDaniel BA, Aske DR. Egg prices, feed costs and the decision to molt. Poult Sci 2000; 79:1242-1245.

13. Anderson KE, Davis GS, Jenkins PK, and Carroll AS. Effects of Bird Age, Density, and Molt on Behavioral Profiles of Two Commercial Layer Strains in Cages1. Poult Sci 2004; 83:15-23.

14. Koelkebeck KW, Parsons CM, LeeperRW, Moshtaghian J. Effect of protein and methionine levels in molt diets on postmolt performance in laying hens. Poult Sci 1991; 70:2063-2073.

15. Statistical Analysis Systems. SAS $®$ versión 8.2 para Windows. Statistical Analysis Systems Institute. Inc. North Carolina. 2002.

16. Yang Z. Comment on Box - Cox transformations in linear models: large sample theory and test of normality. Canad J Statiist 2000; 30: 235-242.
17. Guía de manejo comercial. Hy-lyne Brown 2009-2011, Iowa, USA: HY-LINE International. [citado 1 Ene 2009]. URL Disponible en: URL http://www.avicol.com. co/descargas2/hlb2009-2011.pdf

18. Bell D. Historical and current molting practices in the US table egg industry. Poult Sci 2003; 82:965-970.

19. Bar A, Razaphkovsky V, Wax E,Malka Y. Effect of Age at Molting on Postmolting Performance. Poult Sci 2001; 80:874-878.

20. Webster AB. Behavior of white leghorn laying hens after withdrawal of feed. Poult Sci 2000; 79(2):192.

21. Galeano LF, SorzaJD, Restrepo LF, Vélez CA; Lopera P. Efecto en el tracto reproductivo, digestivo y pérdida de peso corporal del ave semipesada sometida a descanso. [Tesis de Maestría].Colombia: Universidad de Antioquia, Facultad de Ciencias Agrarias, 2007.

22. Flemming E. Control del tamaño del huevo durante la última fase de producción. World Poult 2005; 21:2-5. [fecha de acceso febrero 26 de 2006]. URL disponible en: http:// www. aviagen.com/docs/(Control\%20 del\%20tama\%C3\%B10\%20del\%20huevo). pdf

23. Park SY, BirkholdSG, Kubena LF, Nisbet DJ, Ricke SC. Effects of High Zinc Diets Using Zinc Propionate on Molt Induction, Organs, and Postmolt Egg Production and Quality in Laying Hens. Poult Sci 2004, 83:24-33. 